\title{
The Relationship between Maternal HemoglobinConcen Tretion and Gestational Diabetes Melitus
}

\author{
Zafari. M*, Aghamohammady. A, Tofighi M, Tahmasebi. H. \\ Midwifery Department. Islamic Azad University Sari Branch. Sari. Iran
}

\begin{abstract}
Relation between Hemoglobin Concentration with GDM .In this Cross sectional study, 100 women ( 50 GDM and 50 non GDM ) Selected randomly. informations had registered. there was meaningful difference between Two group from point of parity, job and education .But Age, Weight, gravity, BMI had meaning ful difference. the mean of Neonatal weigh was not different in Both group Accor ding to our study there is no significant Relation Between mothers Hemoglobin concentration during The first trimester and GDM.
\end{abstract}

Keywords Maternal Hemoglobin, Preg Nancy Out Come, Gestational Diabetes

\section{Introduction}

Ges tational diabetes mellitus (GDM) is defined as carbohydrate in tolerance of varying degrees of Severity with onset or first recognition during pregnancy. The current recommendation is to perform screening test between 24 and 26 weeks of gestation, although there are reports claiminy that between $40 \%$ and $66 \%$ of women with GDM could be detected during early pregnancy. The policy of screening in the third trimester has resulted in a significant number of pregnant women delivering big babies, despite good glycemina control, whereas an early screening for Glucose in tolerance and care has resulted in the reduction of some of the hyperglycemia related complications. (1)

Approximately $4 \%$ of pregnant women in the United States have diabetes. Eighty - aight percent of these women have gestational diabetes mellitus (GDM, 450,000 women per year), and the remaining $12 \%$ have either type $1(12,000)$ or type 2 diabetes $(50,000)$ pregnancy is a diabetogenic condition characterized by insulin resistance with a compensatory increase in $\beta$ - call respost and hyperinsulinemia . the placantal secretion of hormones (progestron, cortisol, placental lactogen, prolactin and growth hormone ) is a major contributor to the insulin resistance, which likely plays arole in ensuring that the fetus has an adequate supply of glucose. Pregnancy in patients with diabetes is associated with an increased incidence of congenital abortions in women with poor glycemic control. (2)

Frequency of congenital malformation among infants of diabetic mothers is estimated to be $6-10 \%$ Factors response-

* Corresponding author:

mandanazafari@iausari.ac.ir (Zafari. M)

Published online at http://journal.sapub.org/nursing

Copyright (C) 2011 Scientific \& Academic Publishing. All Rights Reserved ble for these abnormalities are not fully under stood but there are reports suggested that increased free radical production and antioxidant depletion in diabetic pregnant female may contribute to this risk. (3)

The relation between maternal hematological parameters and pregnancy out comes has been a source of countinuirg controversy. In developed countries, not only maternal anemia but also high hemoglobin concentration during pregnancy has been reported to in crease the risks of unfavorable outcomes such as small - for - gestational - age (SGA) birth, preterm birth, and prenatal death . the association between hemoglobin concentration in early pregnancy, changes in hemoglobin concen tretion duriny pregnsncy, and risk of still birth are not known. (4)

There is now occumulated evidence in the literature that high maternal Hemoglobin is associated with increased incidence of adverse pregnancy out comes which include low birth weight and pre term labor.

In no pregnant population, an association between hemoglobin concen tration with Diabetes Mellitus has been reported before. (5)

\section{Objectives}

We decided to study relationship between maternal Hemoglobin concen tration at the first trimester and occurrence of GDM.

\section{Methods}

Having a view to the past, this study was done by investigating the profiles of women who had gone to health care centers in the city of sari in 2011. 100 profiles were chosen, which had exhaustive information about the mothers in- 
cluding: demographic features, test requested at the first visit and next visits which were completely recorded. Sampling was random.

Criteria of being included in the study: conception with a living fetus amounts of FBS and HB in the first 14 weeks of conception and GCT in weeks 24-28 of conception.

And criteria for being excluded from the study were two types of dangerous pregnancies like having clozen- vascular diseases, and disease of heart, vessels, kidneys', the anomy of tuberculosis cycle, anomie, using anti convulsion medications, varfaline and anti-cancer medication.

By using the questionnaire, pieces of information like age, weight, height, number of conceptions and giving birth, amount of HB, FBS in the first visit, GCT in the ages 24-28 and the infants' weights were gathered. Also the pregnancy diabetes was diagnosed by GCT amounts above 135 in ages 24-28 and the disturbance of ctt.

After gathering the information, the dates were analyzed by spss software. To analyze the data, the description, statistical and deductive methods were used.

\section{Results}

From the population of 100 people in this study, 50 persons had gestational diabetes while the other 50 did not .

According to table 1 there were not any differences between the two groups in the number of giving birth, employment condition and education.

Also differences were meaningful in the two groups in age, weight, number of pregnancies and BMI.

Table 1. Some variable in pregnant women

\begin{tabular}{|c|c|}
\hline Variable & P value \\
\hline Gravity & 0.000 \\
\hline Parity & 0.182 \\
\hline job situation & 0.629 \\
\hline Education & 0.292 \\
\hline BMI & 0.000 \\
\hline
\end{tabular}

The age average in mothers contracted with pregnancy diabetes was 30,24 and it was 22,83 in the witness group $p=$ $0 / 00001$. Among healthy mothers, $100 \%$ were under 34 year old and among mothers having the disease, $74 \%$ were under 34 and $26 \%$ were over 34 years old, therefore in the mistake level of $5 \%$, we can say that according to test, there is a meaningful relation between the mothers' aging and contracting the pregnancy diabetes. Also the differences were meaningful in the number of pregnancy and BMI.

According to table number 2, among the people suffering from pregnancy diabetes, the hemoglobin density was above 13 in $28 \%$ of mothers, $12.4-13$ in $32 \%$ of them and below 11.5 in $14 \%$. So we can say that there is not a meaningful difference between the different levels of hemoglobin contracting and pregnancy diabetes.

Also according to t-test, the comparison between the ages of mothers in the two groups based on hemoglobin density shows that in all densities of hemoglobin's the age average of the two groups have a meaningful difference. In comparing the mother's weights in the groups, only in hemoglobine densities under 11.5, there was not a meaningful difference. According to tests, in mistake level of $1 \%$ in hemoglobine density BMI. There is a meaningful difference in the two groups, but in the level of $5 \%$, the difference was meaningful only in densities of 11.6-12.3 and 12.4-13.

Table 2. Comparison the Age, weight, BMI in Both groups

\begin{tabular}{|c|c|c|c|c|}
\hline $\mathrm{Hb}$ & - & Age & Weight of mother & BMI \\
\hline$<11.5$ & - & 0.042 & 0.175 & 0.056 \\
\hline $11.6-12.3$ & - & 0.001 & 0.005 & 0.006 \\
\hline $12.4-13$ & - & 0.000 & 0.002 & 0.014 \\
\hline$>13$ & - & 0.000 & 0.000 & 0.060 \\
\hline
\end{tabular}

\section{Discussion}

It seems that the most important risk factor for gestational diabetes, is high maternal age at pregnancy with a positive family history and BMI during pregnancy.

The treatment process is expensive, and requires follow-up care which is appropriate for the control and surveillance of the financial burden imposed on the family.

In the present study, the levels of hemoglobin and risk of gestational diabetes showed no significant difference.

In the study conducted by Sir, this relationship was not meaningful.

According to studies done by the researchers, there is a high degree of hemoglobin which can be a predictor of gestational diabetes.

However, maternal factors, gestational age, starting taking iron pills, and weight gain at the beginning of trimester of pregnancy are determinants of hemoglobin in the blood.

In the present study, a meaningful relationship between maternal weight and gestational diabetes exists. In the study conducted by Garshasb and colleagues, increasing the pitch of the occurrence of gestational diabetes was associated with higher levels.

In the present study the relationship between birth weight and maternal age and hemoglobin level was significant.

In this regard, in the study conducted by Faraji, the relationship between hemoglobin concentration and birth weight in the first quarter was meaningful.

Also, Guzel asserted that weight gain during pregnancy is a powerful factor for predicting birth weight

Avdl also believes that there exists an inverse relationship between maternal hemoglobin concentration and birth weight.

In comparison with the weight of infants in both groups, significant differences were seen.

Also, the relationship between hemoglobin concentration and birth weight was not significant.

The relationship between maternal age and birth weight were significant.

However, in relation to birth weight and maternal age and hemoglobin level, despite the dual linear model confirmed 
the error level 5 , the concentration of hemoglobin is minor.

However, the presence of this variable leads to a high correlation with age is in a basic mode.

The study was conducted on the relationship between BMI and the weight of the four levels of hemoglobin. The research showed that at concentrations less than 1.5 and the error level of 5, there was a significant relation.

Also, in the tri-linear model, the relationship between hemoglobin concentration and birth weight with BMI and the variables age, despite approval from the level 5, the concentration of hemoglobin is at the lowest level, and the BMW is at the highest level. In total, the three variables are correlated to high levels.

In total, the three variables are correlated to high levels.

Although, we can say there is a positive relationship between the concentration of hemoglobin, the concentration of less than $11 \mathrm{~m}$ between maternal weight and infant's weight.

\section{Conclusions}

Finally, we can say that based on the logistic regression model, risk factors for gestational diabetes, maternal age and risk Hstd hemoglobin and BMI has a lower level.

\section{REFRENCES}

[1] Balaji V, Balajis, A shalatha S, SheelaS. Suresh S, S eshiah V. A 1c in Gestational Diabetes Mellitus in Asian Indion women. Diabetes Care. 2007: 30 (7): 1865 - 1867

[2] Gorge D, Harris M, Russell D, Wihite M. Diabe tes Managcment and exercise in pregnant patients with Diabetes. Clinical Diabetes. 2003 : 23 (4): 165-168

[3] Chaudhari L, Tandon N, Vaney N, Agarwal. Lipid peroxidetion and Antioxident enzymes in Geststional. Diabetics, In dian J PHYSIOL PHARMACOL. 2003 : 43 (4): 441 - 446

[4] Stephansson O, Paul W. Johansson A, Cnattingius S. Maternal Hemoglobin ConCentration Duriny preynancy and Risk of stillbirth. JAMA. 2000.2(20): 2611-2617

[5] Ebrahimzadeh S, Jabarzadeh S, Khadem N, Vaziri Es. Evaluation of relation between maternal Hemoglobin Con centration at the first trimenter and occurrence of GDm, ofogh Donesh. GMutls Journal, 2008: 14 (3): 28-34

[6] Bastani F, Zarabi R. Correlation of self - efficacy among women with gestational diabetes. HAYAT J. 2010; 16(3-4): 107
[7] Sir. Relation ship of prevalence of Gestational Diabetes Mellitus with Moternul Hemoglobin 2005. JA PI. 53: 11077

[8] Bencaiova G, Krafft A, Burkhardt T, Zimmermann. Hemoglobino pathies, body iron stores and ges tational diabetes mellitus. haematologica the hematology journal. 2005 ؛ 90 (8): 1138

[9] Alamollahy H, kariman N, Hossein panah F, Alavi H. Relation Between Hemoglobin in first trimester and gestational diabetes. iranian $\mathrm{J}$ of Endocrine 48. (persain)

[10] Terence T, Louis Y, Kar- Fai T, Lai - Fong H. Moternal Hemoglobin and Risk of Gesta tional Diabetes Mellitus in Chinese women. 2002 ؛ 99(3): $807-812$

[11] www.medical-science.net/Gynemetrics. The Relationship between Maternal Hemoglobin Concentration, Red Blood Cell count and Hema tocrit and Ges tat ional Diabetes Mellitus in Chinese women

[12] Sharifi F, Ziaee A, Feizi A, Mousavinasab N, Anjomshooa A, Mokhtari $p$, Serum ferritin Cocentration in gestational diabetes mellitus and rislc of Subsequent development of early postpartum diabetes mellitus. Diabetes, Metabolic Syndrom and Obesity: Targets and therapy. 2010: 3: $413-419$. (Persian)

[13] Akbarzadeh M. Alizadeh L, Tabatabai H, RamziM. Relation Maternal fac tro with Hemoglobin in pregnancy. south medical J. 2009: 12 (2): 133 - 141. (persain)

[14] Garshasbi E, Sali Z, Faghih zadeh S, Naghizadeh M. the affect acceleration of BmI in pregnancy on pregnancy out come. Daneshvar medical J. 200916 (77): 1-7. (persain)

[15] Tabandeh A, Kashani E. Relation Between primary BmI and weight gain in pregnancy with Maternal 8 neonatal out come. J Medical science of Gorgan. 2007؛ 9 (1): 20 - 24. (persain)

[16] Faraji R, mirbolok F, Sharemi S Asgharnia M, Joafshani M, Glomzadeh M. Relation Between Maternal Hemoglobin Concentration and $\mathrm{BmI}$ duriny the first trimester in primiparous women and her neonatal s Bir th weight. 2010: 18 (1): $62-68$ (persain)

[17] Geutzel L, Zamora Y, Wilkins I, Greisinger A, pregnancy out comes in women with Normal BmI AND WEIGHT GAIN OUTSIDE Recommen dation. Inter national Journal of Gyneacology 8 obs tetrics. 2009 ؛99 (4): 735

[18] Odul A, Amburgey M , Ing E, Gary J , Badger M, Berrstein M, MATERNAL Hemoglobin Concentration and its Association with Birth weight in Newborns of Mothers with preeclam psia. J Matern Fetal Neonatal Med. 2009 ؛ 22 (9): 740-744

[19] Cogswell M, parvanta I, Ickes L, Yip R, Bittenham M . Iron Supplemen tation during preg nancy, anemia and birth weight: a randomized Controlled trial. Am J clin Nutr. 2003 : 78: 773 $-781$ 